\title{
Nueva evidencia taxonómica de los pequeños mamíferos de la Cueva Huenul 1 (Neuquén, Argentina) permite refinar la reconstrucción paleoambiental
}

\author{
Fernando J. Fernández, Juan Pablo Aguilar, Ramiro Barberena y Ulyses F. J. Pardiñas \\ Recibido 19 de abril 2021. Aceptado 21 de julio 2021
}

\begin{abstract}
RESUMEN
Cueva Huenul 1, ubicado en el extremo norte de la provincia del Neuquén, se destaca por un componente paleontológico que incluye megafauna extinta del Pleistoceno tardío ( 16,6 ka cal. AP) y tres componentes arqueológicos que cubren, en forma discontinua, el Holoceno. El sitio presenta restos de micromamíferos en los cuatros componentes. Previamente, se habían registrado 1426 especímenes de pequeños roedores y marsupiales provenientes de la cuadrícula A1. La nueva evidencia analizada corresponde al registro de las cuadrículas B1, C1 y D1 y hace a un total de 1707 especímenes, aspecto que permite refinar las inferencias paleoambientales. Al conjunto faunístico ya conocido para el sitio -conformado por los roedores Akodon iniscatus, Ctenomys sp., Eligmodontia sp., Euneomys chinchilloides, Microcavia australis y Phyllotis xanthopygus y el marsupial Thylamys pallidior-, se adicionan dos nuevos sigmodontinos (Abrothrix olivacea, Oligoryzomys longicaudatus) y un caviomorfo (Galea leucoblephara). Los datos novedosos no favorecen una mayor heterogeneidad ambiental hacia el Holoceno temprano ( 9,5 ka cal. AP) como se había conjeturado previamente. Antes bien, permiten inferir una marcada estabilidad del paisaje en general y, por lo tanto, de las condiciones climáticas, durante el segmento cronológico representado.
\end{abstract}

Palabras clave: Holoceno; Pleistoceno tardío; Noroeste patagónico; Marsupiales; Roedores.

\section{New taxonomic evidence for the small mammals from Cueva Huenul 1 (Neuquén, Argentina) allows refining the palaeoenvironmental reconstruction}

\begin{abstract}
Cueva Huenul 1 site, located in the extreme north of Neuquén Province, is notable for a paleontological component with extinct megafauna in the Late Pleistocene ( 16.6 ka cal BP) and three archaeological components that cover, discontinuously, the Holocene. The site presents small mammals in the four components. Previously, 1426 well-preserved specimens of small rodents and marsupials had been recorded from excavation unit A1. The new evidence corresponds to the small mammals record from units B1, C1 and D1, with a total of 1707 specimens, enabling better palaeoenvironmental inferences to be made. To the previous known assemblage made up of the rodents Akodon iniscatus, Ctenomys

\footnotetext{
Fernando J. Fernández. Consejo Nacional de Investigaciones Científicas y Técnicas (CONICET), Grupo de Estudios en Arqueometría (GEArq), Paseo Colón 1063, Facultad de Ingeniería, UBA, Ciudad Autónoma de Buenos Aires. Cátedra de Zooarqueología, Facultad de Ciencias Naturales y Museo, Universidad Nacional de La Plata, Buenos Aires. E-mail: fernandezf77@yahoo.com.ar

Juan Pablo Aguilar. Instituto de Ciencias Humanas, Sociales y Ambientales (INCIHUSA-CONICET), Av. Ruiz Leal s/n. Facultad de Filosofía y Letras, Universidad Nacional de Cuyo, Mendoza. E-mail: juanpabloaguilar05@yahoo.com

Ramiro Barberena. CONICET, Laboratorio de Paleoecología Humana, Padre Jorge Contreras I300. Facultad de Ciencias Exactas y Naturales, Universidad Nacional de Cuyo, Mendoza. E-mail: ramidus28@gmail.com

Ulyses F. J. Pardiñas. Instituto de Diversidad y Evolución Austral (IDEAus-CONICET), Boulevard Brown 29I5, Puerto Madryn, Chubut, Argentina. Instituto Nacional de Biodiversidad (INABIO), Quito, Ecuador. E-mail: ulyses@cenpat-conicet.gob.ar
} 
sp., Eligmodontia sp., Euneomys chinchilloides and Microcavia australis, Phyllotis xanthopygus) and the marsupial Thylamys pallidior, two new sigmodontines (Abrothrix olivacea, Oligoryzomys longicaudatus) and one caviomorph (Galea leucoblephara) are added. The new data do not favor greater environmental heterogeneity towards the early Holocene ( $9.5 \mathrm{ka}$ cal BP) as previously suggested. Rather, they support the inference of a marked stability of the landscape in general and, therefore, of climatic conditions during the recorded periods.

Keywords: Holocene; Late Pleistocene; Northwestern Patagonia; Marsupials; Rodents.

\section{INTRODUCCIÓN}

En la última década se hicieron numerosas prospecciones y excavaciones arqueológicas en el extremo norte de la provincia del Neuquén, Argentina (e.g., Gordón et al., 2017 y trabajos allí citados). El sitio Cueva Huenul 1 (en adelante $\mathrm{CH} 1$ ) se destaca por las excelentes condiciones de preservación de sus depósitos estratificados, la presencia de un componente paleontológico con megafauna extinta del Pleistoceno tardío ( 16,6 ka cal. AP) y de tres componentes arqueológicos holocénicos (Barberena et al., 2010, 2015; Pompei et al., 2012; Barberena, 2014, 2017). CH1 aportó datos valiosos en materia de aprovisionamiento y contexto artefactual lítico, arte rupestre, registro arqueobotánico, polen, paleoparasitología, isótopos estables y zooarqueología (Beltrame et al., 2011; Fernández et al., 2011a, 2012; Pompei et al., 2012; Llano y Barberena, 2013; Barberena et al., 2015, 2018; Llano et al., 2019, 2020). Algunas de las investigaciones mencionadas se orientaron hacia el análisis paleoambiental (Fernández et al., 2011a, 2012; Pompei et al., 2012; Llano et al., 2020), principalmente para evaluar si los cambios del ambiente tuvieron incidencias en las ocupaciones humanas (Barberena et al., 2015, 2018; Barberena, 2017; Llano et al., 2020).

Previamente, en $\mathrm{CH} 1$ se habían registrado restos bien preservados de micromamíferos provenientes de la cuadrícula A1 (Fernández et al., 2011a, 2012). El estudio de esa muestra permitió establecer un conjunto faunístico conformado por dos taxones de roedores caviomorfos (Ctenomys sp., Microcavia australis), cuatro de roedores sigmodontinos (Akodon iniscatus, Eligmodontia sp., Euneomys chinchilloides, Phyllotis xanthopygus) y un marsupial (Thylamys pallidior). Los restos de estas especies habrían sido acumulados por la lechuza de campanario Tyto furcata (Fernández et al., 2012). La estructura taxonómica general de los conjuntos de micromamíferos se mantuvo estable desde la transición Pleistoceno-Holoceno hasta el Holoceno tardío final. La interpretación paleoambiental efectuada sugirió un paisaje regional de áreas abiertas y rocosas, aunque con mayor heterogeneidad hacia el Holoceno temprano $(\sim 9,5 \mathrm{ka}$ cal. AP), reflejada por el registro de $A$. iniscatus y E. chinchilloides (Fernández et al., 2011a, 2012). En esos trabajos se enfatizó en la necesidad de aumentar la muestra para robustecer la hipótesis paleoambiental.

Afortunadamente, las nuevas excavaciones permitieron recuperar materiales apreciables. En este trabajo se incorporan al análisis los restos de micromamíferos $(<1 \mathrm{~kg})$ de tres cuadrículas inéditas, que abarcan la totalidad del material recuperado. El objetivo principal de esta investigación es brindar un panorama refinado de la estructura taxonómica y del valor paleoambiental de los pequeños roedores y marsupiales de $\mathrm{CH} 1$. Esto permitió rediscutir hipótesis ya formuladas (Fernández et al., 2011a, 2012) y robustecer las nuevas inferencias con otros proxies publicados para el sitio arqueológico (Llano et al., 2020).

\section{MATERIALES Y MÉTODOS}

\section{Sitio arqueológico y marco ambiental}

$\mathrm{CH} 1$ (36 $57^{\circ} \mathrm{S}, 69^{\circ} 49^{\prime} \mathrm{O}, 1000$ msnm; Figura 1A) se ubica en el extremo norte de la provincia del Neuquén, sobre la margen sur del río Colorado, en cercanías de su nacimiento. El sitio se emplaza en un área con extensa actividad volcánica en el retroarco Andino de la Payunia sur, concretamente, dentro del complejo del Tromen (e.g., Folguera et al., 2008). En pocas decenas de kilómetros en línea recta desde $\mathrm{CH} 1$, los rangos altitudinales oscilan desde 850 a 3900 msnm. Esta heterogeneidad topográfica determina que en distancias cortas se verifiquen cambios abruptos en las comunidades de micromamíferos (Fernández, 2012; Fernández et al., 2012; Bernardis, 2019; López et al., 2021). En este sector de Patagonia, comprendido entre los $30^{\circ}-40^{\circ} \mathrm{S}$, ocurre la transición de los sistemas de circulación atmosférica Subtropical y Templado, lo que se traduce en un clima árido y semiárido, con 
una variabilidad en la estacionalidad de las precipitaciones, que oscilan entre 200 y 300 mm anuales. La vegetación incluye un mosaico de comunidades fitogeográficas del desierto arbustivo del Monte y de la estepa arbustiva y herbácea Patagónica y Altoandina (e.g., Abraham et al., 2009).

Se realizaron dos campañas de excavación en CH1: en 2009 se abrieron las cuadrículas A1 $(2 \times$ $1 \mathrm{~m})$ y B1 $(1 \times 1 \mathrm{~m})$ y en 2012 , las cuadrículas C1 y D1 $(2 \times 1 \mathrm{~m}$ cada una). Esto totaliza una superficie excavada de $7 \mathrm{~m}^{2}$ y un volumen de sedimento removido de $7,75 \mathrm{~m}^{3}$ (Barberena, 2014, 2017). Las cuadrículas A1, C1 y D1 se ubican en posición adyacente y comparten, en términos generales, la misma secuencia estratigráfica. La cuadrícula B1 se localiza en el sector más externo de la cueva y solo presenta el segmento temporal más reciente (Figura 1B).

La estratigrafía del sitio se organizó en cuatro componentes cronoestratigráficos que se extienden entre ca. 16,6 y 0,4 ka cal. AP (las fechas fueron publicadas en detalle, ver Barberena, 2014, 2017): el Componente 1 abarca el Pleistoceno final (16,6-13,6 ka cal. AP) y carece de evidencias arqueológicas in situ. Su límite superior está marcado por una discordancia erosiva denominada Hiato 1 (Figura 1C). El Componente 2 se extiende entre 11,3-10,2 ka cal. AP (Figura 1C). En los niveles que lo integran se registraron las primeras ocupaciones humanas. El Componente 3 corresponde en su totalidad a la estructura sedimentaria antrópica $G$, que pertenece a un amplio pozo en cubeta relleno de material vegetal (Senna aphylla) cubierto por ocre rojo y datado en 5,6-5,3 ka cal. AP (Llano et al., 2019). Hasta el momento, representa la única evidencia del Holoceno medio en $\mathrm{CH} 1$. Finalmente, el Componente 4 se registra en todas las cuadrículas excavadas con fechados de 1,50,4 ka cal. AP, que lo vinculan al Holoceno tardío final (Figura 1C). Este coincide con un pulso de elevada ocupación regional y, en la escala del sitio, se asocia a las evidencias antrópicas más abundantes y diversas (Barberena et al., 2015; Llano et al., 2019).

En trabajos previos, Fernández y coautores (2011a, 2012) estudiaron la muestra de micromamíferos recuperada de la cuadrícula A1 (Número de Especímenes Identificados por Taxón, NISP = 1426; Número Mínimo de Individuos, $\mathrm{MNI}=87$ ). Las nuevas muestras aquí incorporadas corresponden a los restos de micromamíferos $($ NISP $=1707)$ de las cuadrículas B1 (NISP = 203), C1 (NISP = 921) y D1 (NISP = 583). En este trabajo se considerará la muestra total de $\mathrm{CH} 1$ (cuadrículas A1, B1, C1 y D1), conformada por un NISP = 3135 y un $\mathrm{MNI}=324$.

Los resultados tafonómicos de las cuadrículas B1, C1 y D1 se expresaron en el trabajo de Fernández y coautores (2017), los cuales coinciden con aquellos presentados por Fernández y coautores (2011a, 2012) para la cuadrícula A1. En síntesis, los
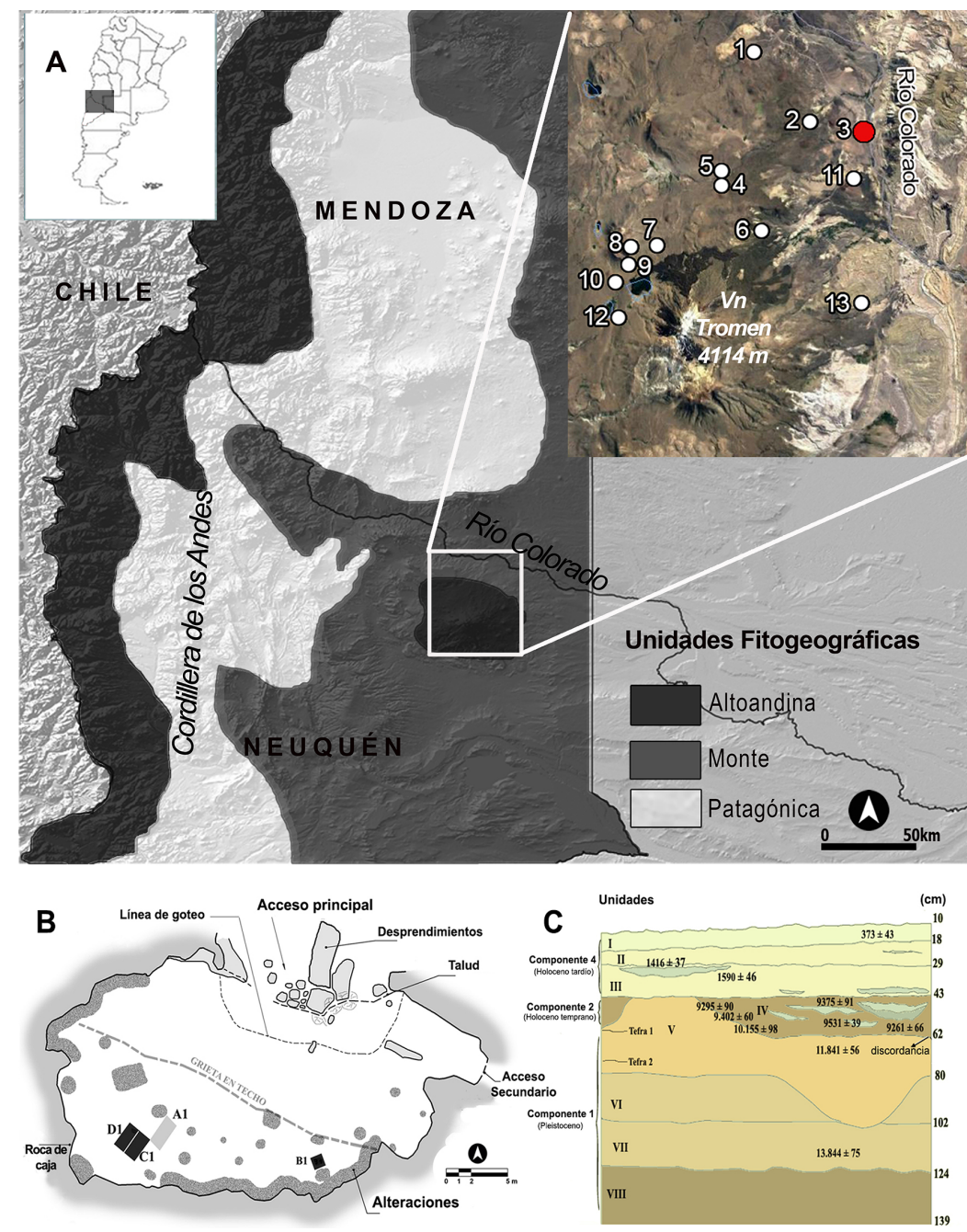

Figura 1. A. Ubicación del sitio arqueológico Cueva Huenul 1 (círculo rojo) y las muestras actuales de egagrópilas de aves Strigiformes (círculos blancos con numeración indicada en la nota de la Tabla 1); B. Planta del sitio; C. Perfil estratigráfico, esquemático. 
bajos valores de corrosión digestiva registrados en todos los componentes -principalmente del tipo ligera, acorde con las distintas morfologías dentarias de los grupos de micromamíferos representados en el sitiosugieren que los restos fueron acumulados por aves Strigiformes, posiblemente Tyto furcata (Fernández et al., 2017, pp. 45-46). La representación de roedores en los conjuntos con señal antrópica es mínima y el foco habría estado en el consumo de Lama guanicoe y especies vegetales (Maihueniopsis, Prosopis, etc.). En este sentido, no hay una evidencia clara de ampliación de la dieta mediante la incorporación de recursos animales de bajo ranking (Barberena et al., 2015; Llano et al., 2019).

\section{Métodos}

Los restos de micromamíferos se examinaron con lupa binocular y las determinaciones taxonómicas se realizaron comparando los restos craneodentarios con ejemplares de referencia de la colección de mamíferos del Grupo de Estudios en Arqueometría de la Facultad de Ingeniería (Universidad de Buenos Aires) y bibliografía (e.g., Fernández et al., 2011b).

Para el análisis paleoambiental, se evaluaron la presencia/ausencia y los cambios en las frecuencias relativas calculadas a partir del MNI de algunas especies de micromamíferos consideradas como indicadores. La aproximación paleoecológica se basa, principalmente, en los requerimientos ambientales y de distribución conocidos para las distintas especies. Este método está afectado por la historia tafonómica de los agregados y el tamaño de la muestra (e.g., Andrews, 1990; Pardiñas, 1999). Como parámetro actualista se utilizaron muestras de egagrópilas de $T$. furcata recuperadas en el sitio y en zonas adyacentes (Tabla 1, Figura 1A).

\begin{tabular}{|c|c|c|c|c|c|c|c|c|c|c|c|c|c|c|c|c|c|}
\hline 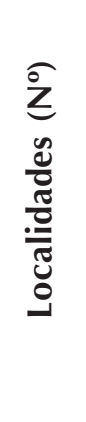 & 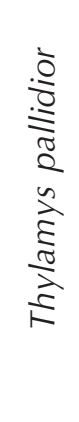 & 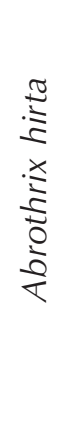 & 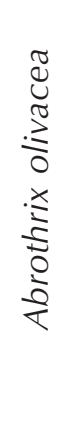 & 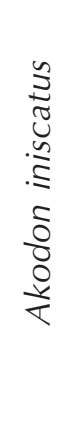 & 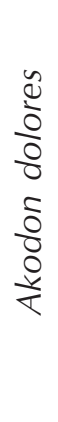 & 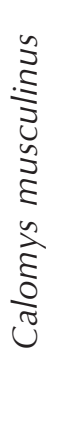 & 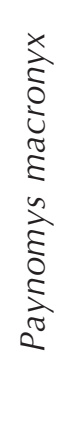 & 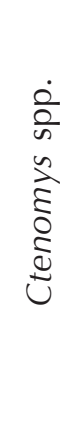 & 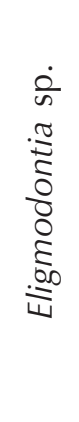 & 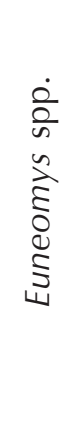 & 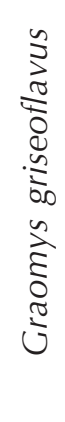 & 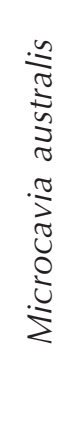 & 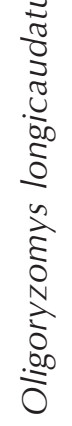 & 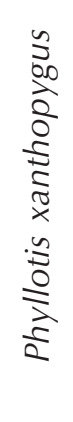 & 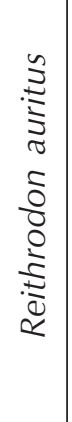 & 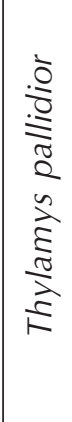 & 吾 \\
\hline 1 & 14 & 0 & 0 & 2 & 0 & 0 & 0 & 25 & 34 & 0 & 1 & 6 & 0 & 23 & 2 & 14 & 121 \\
\hline 2 & 0 & 0 & 0 & 0 & 0 & 0 & 0 & 22 & 0 & 1 & 0 & 1 & 0 & 2 & 1 & 2 & 29 \\
\hline 3 & 0 & 0 & 0 & 0 & 0 & 1 & 0 & 0 & 7 & 0 & 0 & 4 & 0 & 2 & 0 & 0 & 14 \\
\hline 4 & 1 & 0 & 0 & 0 & 0 & 0 & 0 & 0 & 1 & 0 & 0 & 0 & 0 & 2 & 0 & 0 & 4 \\
\hline 5 & 0 & 0 & 1 & 0 & 0 & 0 & 0 & 6 & 2 & 7 & 0 & 2 & 0 & 4 & 1 & 0 & 23 \\
\hline 6 & 10 & 4 & 5 & 9 & 0 & 0 & 0 & 31 & 76 & 11 & 0 & 17 & 1 & 62 & 4 & 10 & 240 \\
\hline 7 & 0 & 9 & 12 & 0 & 0 & 0 & 2 & 31 & 37 & 50 & 0 & 0 & 0 & 1 & 1 & 0 & 143 \\
\hline 8 & 0 & 5 & 7 & 0 & 0 & 0 & 5 & 69 & 12 & 39 & 0 & 0 & 0 & 1 & 2 & 0 & 140 \\
\hline 9 & 0 & 3 & 7 & 0 & 0 & 0 & 11 & 50 & 13 & 41 & 0 & 0 & 0 & 1 & 0 & 0 & 126 \\
\hline 10 & 0 & 0 & 1 & 0 & 0 & 0 & 0 & 2 & 1 & 4 & 0 & 0 & 0 & 0 & 0 & 0 & 8 \\
\hline 11 & 31 & 0 & 0 & 15 & 0 & 0 & 0 & 46 & 41 & 0 & 0 & 15 & 1 & 26 & 23 & 31 & 229 \\
\hline 12 & 0 & 3 & 2 & 0 & 0 & 0 & 8 & 53 & 23 & 27 & 0 & 1 & 1 & 0 & 1 & 0 & 119 \\
\hline 13 & 55 & 0 & 0 & 9 & 1 & 2 & 0 & 37 & 74 & 2 & 9 & 13 & 7 & 22 & 7 & 27 & 265 \\
\hline otal & 111 & 24 & 35 & 35 & 1 & 3 & 26 & 372 & 321 & 182 & 10 & 59 & 10 & 146 & 42 & 84 & 1461 \\
\hline
\end{tabular}

Nota: Muestras actuales de egagrópilas de Strigiformes empleadas como parámetro actualístico. Las localidades 3 ( $36^{\circ} 57^{\prime} \mathrm{S}$, $\left.69^{\circ} 49^{\prime} \mathrm{O}, 1000 \mathrm{msnm}\right)$ y 4 ( $\left.36^{\circ} 59^{\prime} 19,08^{\prime \prime} \mathrm{S}, 6^{\circ} 59^{\prime} 51,06^{\prime \prime} \mathrm{O}, 1813 \mathrm{msnm}\right)$ son novedosas. Las localidades restantes fueron reportadas en Fernández y coautores (2012, p. 24). Localidades: 1= Barrancas; $2=$ Buta-Có, P.P. Tromen; 3= Cueva Huenul actual; 4= Paso de las Vueltas 4; $5=$ P.P. Tromen Arroyo; $6=5,5 \mathrm{~km} \mathrm{~W}$ Buta Ranquil; $7=12 \mathrm{~km} \mathrm{NE}$ refugio P.P. Tromen; $8=$ Cerro Wayle; $9=$ P.P. Tromen; $10=2,2 \mathrm{~km}$ W Laguna Tromen; $11=$ Ea. Corcel Negro; $12=4 \mathrm{~km}$ SW Laguna Tromen; $13=12 \mathrm{~km}$ SE Buta Ranquil.

Tabla 1. Muestras actuales empleadas como parámetro actualista. 
Se calcularon las curvas de rarificación para las muestras arqueológicas y actuales para evaluar la confiabilidad de la riqueza (NTaxa) y la abundancia taxonómica en términos de MNI. Además, se hicieron curvas de rarificación en base al índice de Shannon $\left(\mathrm{H}^{\prime}\right)$ y al $\mathrm{MNI}$, para ajustar las comparaciones entre muestras en materia de diversidad taxonómica. Se realizó un análisis de correspondencias para explorar el ordenamiento de las especies y de las muestras arqueológicas y actuales en forma conjunta, a través de una matriz de valores de abundancia (MNI transformados a logaritmo natural). Los análisis estadísticos se realizaron mediante el uso del programa PAST (PAleontologicalSTatistics), versión 4.03 .

\section{RESULTADOS}

A partir del material recuperado de todas las cuadrículas de $\mathrm{CH} 1$, la composición y abundancia taxonómica de los micromamíferos por cada componente arqueológico se detalla en la Tabla 2. El roedor sigmodontino Eligmodontia sp. es dominante en todos los componentes, con valores que oscilan entre $\sim 40$ y $47 \%$ del $\mathrm{MNI}$ total. Continúan, en orden de abundancia $(\sim 5-20 \%)$ y con proporciones relativamente constantes a lo largo de la secuencia, los roedores caviomorfos Ctenomys sp. y Microcavia australis, el sigmodontino Phyllotis xanthopygus y el marsupial Thylamys pallidior. Estos taxones, a su vez, tienen alta representación en las muestras

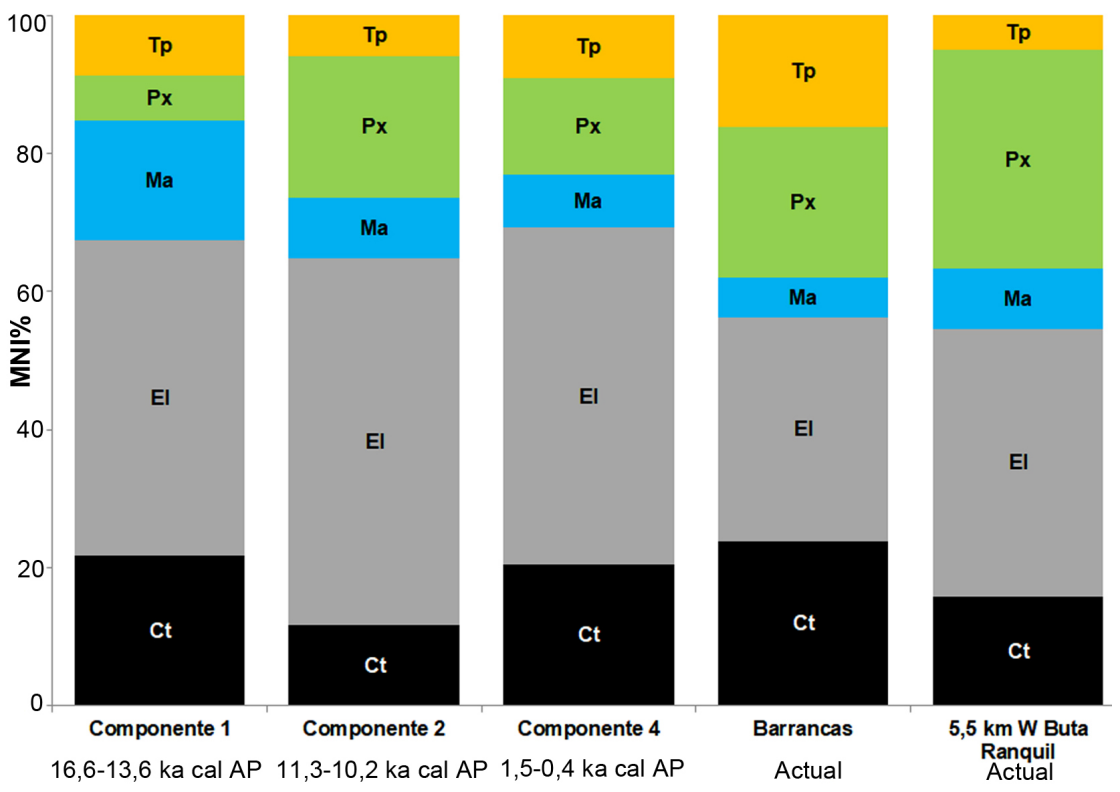

Figura 2. Cambios temporales en la abundancia relativa de algunas especies de micromamíferos en el sitio Cueva Huenul 1. Se excluyó al Componente 3 por tener un $\mathrm{MNI}=4$. Referencias: El: Eligmodontia sp., Ct: Ctenomys sp., Ma: Microcavia australis, Px: Phyllotis xanthopygus, Tp: Thylamys pallidior. actuales de egagrópilas de Tyto furcata recolectadas en cercanías de CH1 (Figura 2). En líneas generales, se mantienen las mismas tendencias taxonómicas previamente reseñadas para el sitio sobre la base del material de la cuadrícula A1, si bien con la incorporación de los restos de las cuadrículas B1, C1 y D1 algunos componentes se enriquecen con el caviomorfo Galea leucoblephara (Componentes 1, 2 y 4) y los sigmodontinos Abrothrix olivacea (Componente 1) y Oligoryzomys longicaudatus (Componentes 1 y 4). Por otra parte, los sigmodontinos Akodon iniscatus y Euneomys chinchilloides, previamente restringidos al Holoceno, a partir de este estudio se extienden también al Pleistoceno tardío (Tabla 2).

El análisis de rarificación realizado sobre los valores de riqueza y diversidad taxonómica (índice de Shannon) muestra que el conjunto arqueológico de micromamíferos del Componente 4 (1,5-0,4 ka cal. $\mathrm{AP})$ es el único que alcanza una asíntota. De esta forma, constituye el componente estadísticamente más significativo, pero dificulta su comparación con los otros componentes arqueológicos (Figura 3). La mayor parte de las muestras de micromamíferos actuales de egagrópilas de $T$. furcata, con un $\mathrm{MNI}>50$, tienen un comportamiento con tendencia asintótica y constituyen una herramienta confiable como parámetros actualistas, considerando la riqueza (Figura 3A) y la diversidad taxonómica (Figura 3B). En este sentido, la Figura 4A grafica los valores de riqueza y diversidad de los componentes arqueológicos y de las muestras actuales más robustas, y señala además escasas variaciones entre ellas $y$, principalmente, entre los componentes arqueológicos y las muestras actuales más cercanas al sitio (e.g., Barrancas).

En congruencia con los resultados reseñados, el análisis de correspondencias (Figura 4B) exhibe un agrupamiento de las muestras arqueológicas y las actuales más próximas al sitio, lo que sugiere una estabilidad de las comunidades de micromamíferos a lo largo del tiempo. El Eje 1 (62,1\% de la varianza total) agrupa, hacia los valores negativos, 


\begin{tabular}{|c|c|c|c|c|c|c|c|c|}
\hline Cronología (en ka, cal. AP) & \multicolumn{2}{|c|}{$1,6-0,4$} & \multicolumn{2}{|l|}{5,5} & \multicolumn{2}{|c|}{$13,6-10,4$} & \multicolumn{2}{|l|}{16,8} \\
\hline Componente & \multicolumn{2}{|l|}{4} & \multicolumn{2}{|l|}{3} & \multicolumn{2}{|l|}{2} & \multicolumn{2}{|l|}{1} \\
\hline \multirow{2}{*}{ Unidad } & \multicolumn{2}{|l|}{ I-III } & \multicolumn{2}{|c|}{ (Estr. G) } & \multicolumn{2}{|l|}{ IV } & \multicolumn{2}{|c|}{ V-VIII } \\
\hline & NISP & MNI & NISP & MNI & NISP & MNI & NIPS & MNI \\
\hline \multicolumn{9}{|l|}{ Didelphimorphia } \\
\hline Thylamys pallidior & 37 & 20 & 0 & 0 & 2 & 2 & 4 & 4 \\
\hline \multicolumn{9}{|l|}{ Rodentia } \\
\hline Hystricomorpha indet.* & 336 & - & 0 & - & 33 & - & 34 & - \\
\hline \multicolumn{9}{|l|}{ Ctenomyidae } \\
\hline Ctenomys sp. & 113 & 45 & 0 & 0 & 19 & 4 & 34 & 10 \\
\hline \multicolumn{9}{|l|}{ Caviidae } \\
\hline Galea leucoblephara & 1 & 1 & 0 & 0 & 1 & 1 & 1 & 1 \\
\hline Microcavia australis & 24 & 17 & 2 & 1 & 8 & 3 & 15 & 8 \\
\hline Cricetidae indet.* & 1117 & & 16 & & 358 & & 539 & \\
\hline Akodon iniscatus & 5 & 4 & 0 & 0 & 2 & 1 & 2 & 2 \\
\hline Abrothrix olivacea & 0 & 0 & 0 & 0 & 0 & 0 & 1 & 1 \\
\hline Phyllotis xanthopygus & 57 & 31 & 0 & 0 & 9 & 7 & 3 & 3 \\
\hline Eligmodontia sp. & 256 & 108 & 5 & 3 & 53 & 18 & 40 & 21 \\
\hline $\begin{array}{l}\text { Oligoryzomys } \\
\text { longicaudatus }\end{array}$ & 1 & 1 & 0 & 0 & 0 & 0 & 1 & 1 \\
\hline Euneomys chinchilloides & 3 & 3 & 0 & 0 & 2 & 2 & 1 & 1 \\
\hline Total & 1950 & 230 & 23 & 4 & 487 & 38 & 675 & 52 \\
\hline
\end{tabular}

arqueológicos se relaciona con la abundancia de especies asociadas a ambientes arbustivos, abiertos y rocosos, que pueden habitar tanto en el Monte como en la estepa patagónica (Eligmodontia spp., M. australis, P. xanthopygus, T. pallidior). Esto resulta coherente con la virtualmente nula ocurrencia de elementos típicamente patagónicos en la historia de $\mathrm{CH} 1$ (escasa representación de $A$. olivacea y E. chinchilloides; ausencia de $A$. hirta y $P$. macronyx).

\section{DISCUSIÓN}

*Únicamente restos poscraneanos que posiblemente correspondan a los taxones identificados.

Tabla 2. Micromamíferos registrados en el sitio arqueológico Cueva Huenul 1 (expresado en NISP y MNI).

a los taxones principalmente asociados a la estepa patagónica y, en la topografía local, a mayores niveles de altitud (Abrothrix spp., Euneomys spp., Paynomys macronyx); hacia los valores positivos se concentran las especies que habitan en el Monte y zonas de menor altitud (Akodon dolores, Calomys musculinus, Graomys griseoflavus, G. leucoblephara) o potencialmente comunes a ambos ambientes (A. iniscatus, Eligmodontia spp., M. australis, O. longicaudatus, P. xanthopygus, Reithrodon auritus, T. pallidior). El Eje $2(14,6 \%$ de la varianza total) reúne hacia los valores positivos a los sigmodontinos típicos del Monte (A. dolores, C. musculinus, G. griseoflavus), ausentes en $\mathrm{CH} 1$ y registrados en algunas muestras actuales ubicadas en un rango altitudinal de entre 1000 y 1450 msnm (Figura 1A, Tablas 1 y 2). La posición central y levemente hacia los valores positivos de los componentes
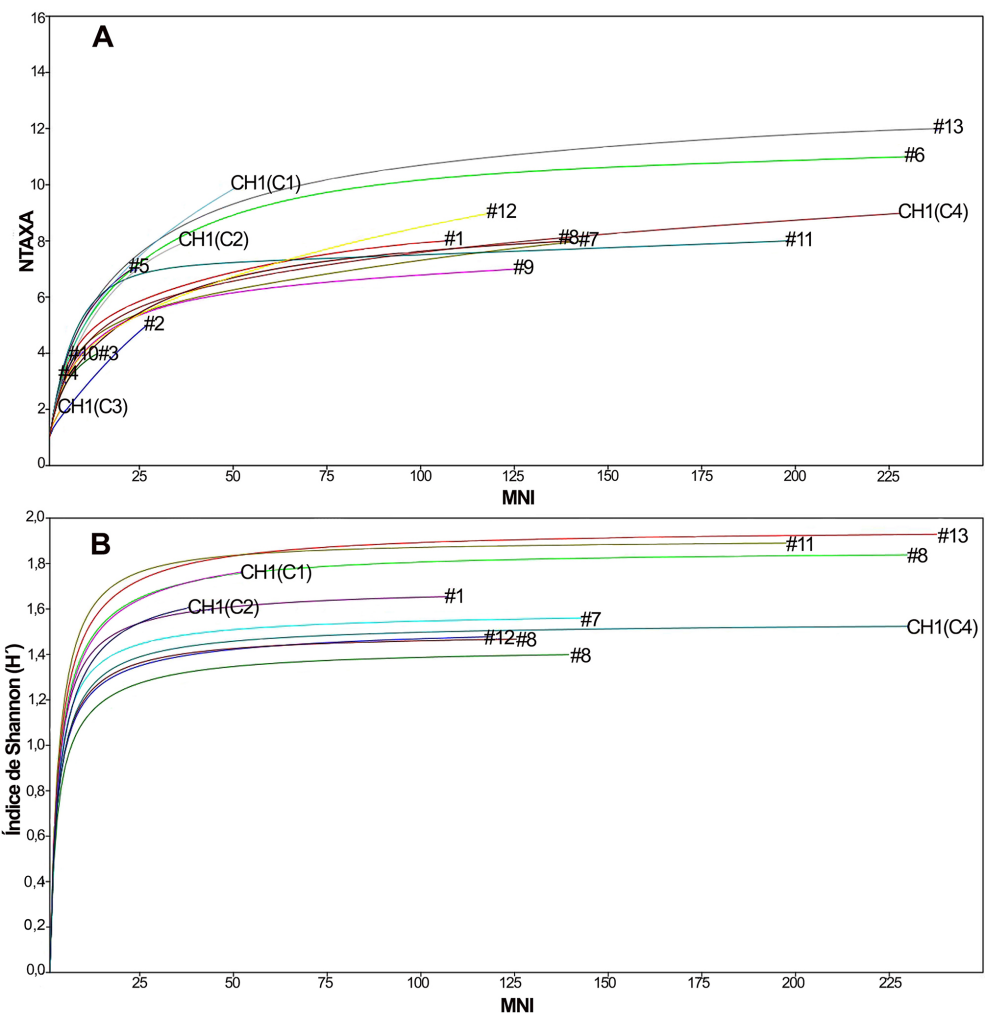

Figura 3. Análisis de rarificación de las muestras de micromamíferos arqueológicas y actuales en base a la riqueza taxonómica $(A)$ y en base al índice de diversidad de Shannon (B). La numeración de las muestras actuales se indicó en la nota de la Tabla 1. 

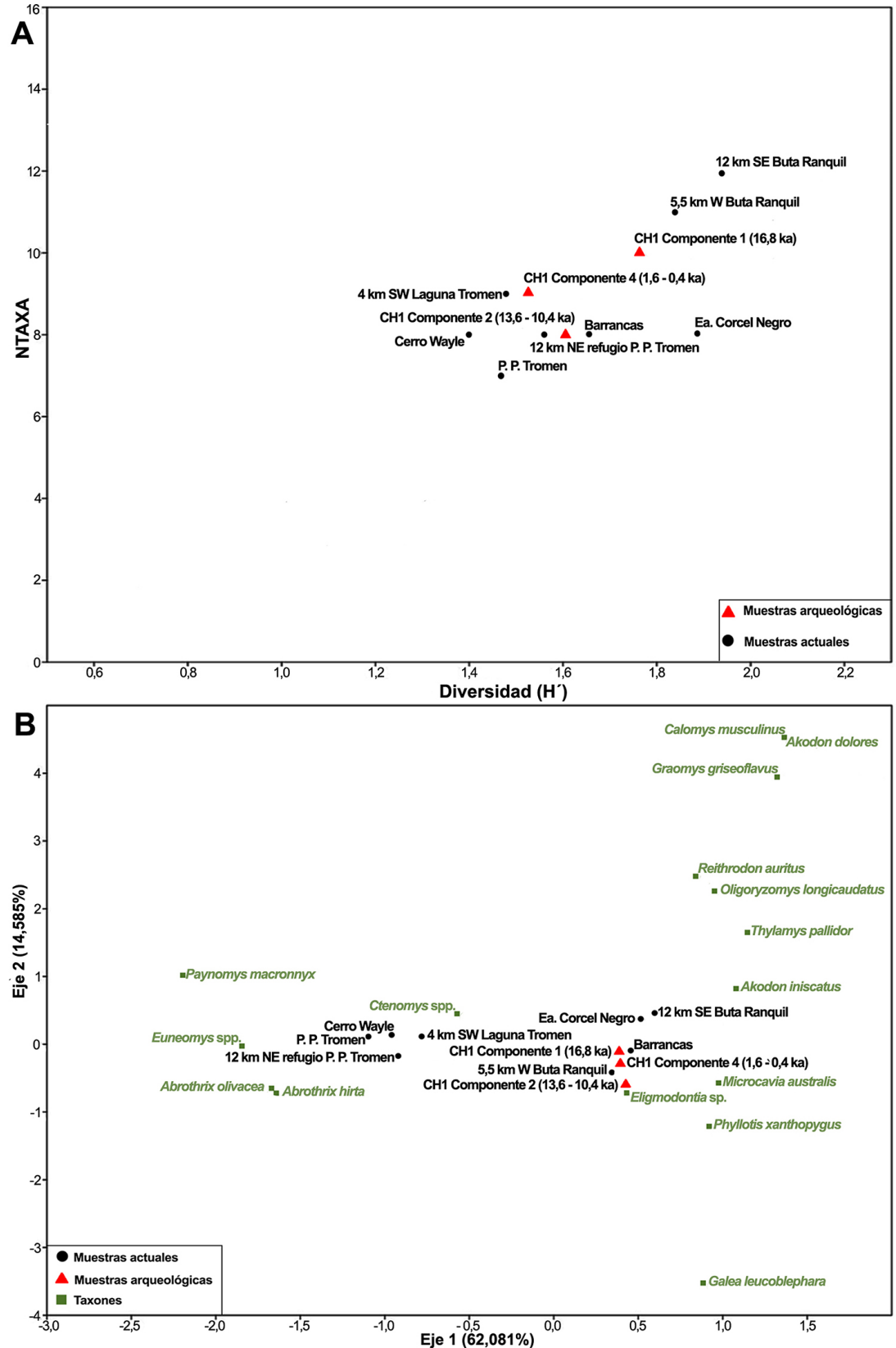

Figura 4. A. Riqueza (NTaxa) e índice de diversidad de Shannon ( $\left.\mathrm{H}^{\prime}\right)$ de las muestras de micromamíferos del sitio Cueva Huenul 1 y de las muestras actuales de Tyto furcata; B. Análisis de correspondencias de las muestras de micromamíferos del sitio Cueva Huenul 1 y de las muestras actuales de egagrópilas de Strigiformes. Ambos ejes muestran los porcentajes de la varianza.

El aumento significativo en la muestra estudiada permite refinar algunos aspectos de la reconstrucción paleoambiental. En primer lugar, es necesario remarcar que la secuencia cronoestratigráfica de $\mathrm{CH} 1$ es discontinua y registra un segmento del Pleistoceno tardío-Holoceno temprano y otro del Holoceno tardío; la evidencia para el Holoceno medio es puntual, y al tratarse de una estructura sedimentaria antrópica, no representaría en forma significativa aportes no humanos. Por lo tanto, el planteo de estabilidad de las comunidades de micromamíferos que aquí se hace debe ser tomado con cautela. Al menos para las ventanas temporales indicadas, la estabilidad de los componentes faunísticos principales, tanto en presencia/ausencia como en abundancias relativas, resulta manifiesta.

El incremento de la muestra estudiada implicó, entre algunas novedades taxonómicas, el registro de Abrothrix olivacea como exclusivo del componente más antiguo. Si bien su presencia está sustentada por un único ejemplar, considerando su ausencia en los demás componentes, merece un tratamiento más pormenorizado. A. olivacea, al igual que el congenérico A. hirta, son abrotriquinos de amplia distribución y notable abundancia tanto en Patagonia continental e insular, andina y extraandina, pero están virtualmente ausentes en las porciones que quedan incluidas en el desierto del Monte (e.g., Lozada et al., 1996; Pardiñas et al., 2003). Esta restricción ambiental resulta elocuente en las muestras de egagrópilas actuales empleadas como parámetro comparativo (Tabla 1). En efecto, A. olivacea es depredado por lechuzas en las partes más altas del gradiente topográfico. Su ocurrencia en el componente finipleistoceno de $\mathrm{CH} 1$ podría indicar un descenso altitudinal de elementos faunísticos. Esto, a su vez, podría ser respuesta a condiciones climáticas más frías que las actuales imperantes en el área, vinculadas con un ambiente posglacial. Este tipo de fenómenos, coherente con englazamientos en los máximos orográficos, ha sido conjeturado en la cuenca superior del río Limay para explicar el registro fósil de Euneomys mordax en Cueva Traful 1 y Cueva del Caballo (Pearson, 1987; Pearson y 
Pearson, 1993; Tammone et al., 2016).

Los análisis realizados apuntan en definitiva a que, aunque los taxones ya estaban presentes en el Pleistoceno tardío, sus abundancias parecen ser un poco diferentes. En el área general de $\mathrm{CH} 1$ y en la zona adyacente boreal de la cuenca media del río Grande, la penetración de elementos más típicos del Monte (Akodon dolores, Galea leucoblephara o Graomys griseoflavus) parece verificarse siguiendo los principales cursos de los ríos y sus áreas de influencia (Fernández et al., 2015a). Contra eso, en tierras "intermedias" entre los valles fluviales y las porciones altoandinas, parece que las comunidades están conformadas por estos taxones (Eligmodontia, Microcavia, Phyllotis, Thylamys) y que la clave de su expresión paleoambiental podría intentar buscarse en sus abundancias, antes bien que en sus presencias.

\section{Integración con otros proxies paleoambientales}

La información paleoambiental del Pleistoceno tardío-Holoceno sigue siendo pobre en el extremo norte del Neuquén, concentrada en registros polínicos, arqueobotánicos, antracológicos y en paleomadrigueras de roedores (e.g., Markgraf et al., 2009; Fernández et al., 2011a, 2012; Pompei et al., 2012; Llano et al., 2019, 2020). Si ampliamos el área al noroeste de Patagonia y a ambas vertientes de los Andes, resulta una abundancia y diversidad mayor de proxies paleoambientales, con la adición de registros glaciológicos, sedimentológicos y dendrocronológicos (e.g., Mercer, 1982; Markgraf, 1983; Jenny et al., 2002; Espizua, 2003, 2005; Hajdas et al., 2003; Hofreiter et al., 2003; Gil et al., 2005; Whitlock et al., 2006; Espizua y Pitte, 2009; Bianchi y Ariztegui, 2012; de Jong et al., 2013; FrugoneÁlvarez et al., 2020). En este panorama, los análisis que involucran restos de micromamíferos sugieren gran potencial, aunque por ahora la indagación sobre este tipo de materiales ha sido escasa (e.g., Pearson, 1987; Pardiñas, 1999; Teta et al., 2005, 2014; Agnolín y Pérez, 2014; Fernández et al., 2015a, 2015b, 2016; Fernández, 2012; Pardiñas y Teta, 2013; Tammone et al., 2016; Fernández y Pardiñas, 2018; López et al., 2021).

El análisis integral de los restos de micromamíferos de $\mathrm{CH} 1$ corrobora la hipótesis paleoambiental planteada a partir de los restos de la cuadrícula A1: un escenario de estabilidad ambiental desde el Pleistoceno tardío hasta la actualidad (Fernández et al., 2011a, 2012). En este contexto y desde los 16,6 ka cal. AP, los conjuntos locales habrían estado dominados por especies de micromamíferos que hoy en día habitan en áreas abiertas, arbustivas y rocosas de baja a media altitud, tanto en el desierto del Monte como en la estepa patagónica. Sin embargo, la discordancia erosiva revelada en $\mathrm{CH} 1$ entre 11,8 y 9,5 cal. AP (Barberena et al., 2010, 2015; Barberena, 2014, 2017) pudo ocultar los efectos del aumento de la aridez y del enfriamiento ocurridos a finales del Pleistoceno, en ocasión de la Inversión Climática Huelmo Mascardi (Hajdas et al., 2003). Este cambio climático tuvo una mayor expresión en otros sectores del noroeste de Patagonia, con variaciones en la composición florística local y/o el incremento en los regímenes de incendios forestales detectados en los bosques de Austrocedrus y Nothofagus (Whitlock et al., 2006; Bianchi y Ariztegui, 2012). Debido a la discordancia erosiva, ningún componente de $\mathrm{CH} 1$ tiene dataciones sincrónicas con los eventos áridos detectados en la región para el Holoceno medio (e.g., Markgraf, 1983; Gil et al., 2005; Zárate et al., 2010; Frugone-Álvarez et al., 2020; Llano et al., 2020). En un mismo sentido, los depósitos de $\mathrm{CH} 1$ tampoco se pueden correlacionar con los neoavances glaciarios registrados en los valles El Azufre y El Peñón del sistema del río Valenzuela de la cuenca superior del río Grande durante el Holoceno medio (5,7-4,4 ka cal. AP) y el Holoceno tardío (2,5-2,2 ka cal. AP), incluso, en tiempos más recientes $(0,4-0,15$ ka cal. AP) vinculados con la Pequeña Edad de Hielo (Mercer, 1982; Espizua, 2003, 2005; Espizua y Pitte, 2009). Sin embargo, se obtuvieron dataciones radiocarbónicas asociadas al Holoceno temprano y medio a partir de los excrementos del chinchillón Lagidium (Rodentia, Chinchillidae) recuperados de paleomadrigueras halladas en la localidad de $\mathrm{CH}$ (Llano et al., 2020). A partir de los macrorrestos vegetales, del polen y de los parásitos contenidos en esas deposiciones, se registró un aumento de la aridez entre 9,2 y 5,5 ka cal. AP. La escasez de restos de micromamíferos en la estructura antrópica $\mathrm{G}$ de $\mathrm{CH} 1$, con cronología asociada al Holoceno medio (5,6-5,3 ka cal. AP), tal vez sea la razón por la cual no se registraron las especies típicas del Monte (e.g., Akodon dolores, Calomys musculinus, Graomys griseoflavus) que están presentes en algunas muestras actuales recolectadas en cercanías del sitio.

A pesar de estas limitaciones cronoestratigráficas, se plantea un ambiente local estable que 
incluye un mosaico de estepas arbustivas, áreas abiertas con suelo desnudo y pedregoso, algo menos árido que en la actualidad. En coincidencia, el estudio de los granos de polen recuperados de los componentes estratigráficos de $\mathrm{CH} 1$ indicó el desarrollo de una estepa arbustiva y herbácea sin mayores cambios a lo largo de la secuencia (Pompei et al., 2012). Entre 13,8 y 9,5 ka cal. AP, el registro polínico de arbustos (Lycium, Schinus), arbustos en cojín (Ephedra, Nassauvia) y gramíneas que actualmente se encuentran a $1000 \mathrm{~m}$ por encima de $\mathrm{CH} 1$ indicó la presencia de estepas arbustivas y graminosas típicas de la estepa patagónica. Para este segmento temporal, este proxy paleoambiental expuso condiciones locales más frías que la actualidad, algo no manifiesto en el registro de micromamíferos. Mayor coincidencia entre ambos tipos de indicadores paleoambientales se observa entre 1,6 y $0,4 \mathrm{ka}$ cal. AP, donde domina el polen de arbustos (e.g., Lycium, Schinus) y disminuye la proporción de gramíneas, lo que sugiere el desarrollo de una estepa arbustiva-graminosa de ecotono Monte y estepa patagónica similares a la actualidad (Pompei et al., 2012). Otras muestras de polen y de macrorrestos vegetales recuperadas de las heces de Lagidium provenientes de paleomadrigueras de $\mathrm{CH}$ cubren algunos períodos cronológicos no detectados en los componentes estratigráficos (Llano et al., 2020). Durante el Holoceno temprano (10,5-9,4 ka cal. AP), dominan los arbustos del Monte tales como Larrea, Lycium y Prosopis, junto con arbustos de la estepa patagónica (e.g., Adesmia, Berberis) y con arbustos del ecotono Monte-Patagonia (Ephedra, Schinus, Senecio). Las condiciones de aridez se intensifican hacia el 9,2 ka cal. AP y durante el Holoceno medio (6,5-5,5 ka cal. AP), cuando la vegetación del Monte (e.g., Larrea, Prosopis) prevalece sobre la vegetación patagónica y de transición entre ambos biomas. Hacia el Holoceno tardío, expresado en los intervalos 4,23,8 y 3,5-2,5 ka cal. AP, la codominancia de arbustos del Monte (Larrea, Lycium, Prosopis), arbustos y gramíneas patagónicos (e.g., Baccharis, Chuquiraga, Festuca, Jarava, Mulinum, Nassauvia, Poa) y arbustos del ecotono Monte-Patagonia (Ephedra, Schinus y Senecio) sugiere condiciones de humedad ligeramente mayores que en la actualidad (Llano et al., 2020).

Por otra parte, la estabilidad ambiental indicada para los componentes estratigráficos de $\mathrm{CH} 1$ coincide con el registro polínico de Mallín Vaca Lauquen para el segmento temporal de 17,8-8,9 ka ${ }^{14} \mathrm{C}$ AP (Markgraf et al., 2009) y con los datos proporcionados por los excrementos de Lagidium recuperados de las paleomadrigueras del sitio Cuchillo Curá (centro del Neuquén) hacia 16,4 ka cal. AP (Hofreiter et al., 2003). Diversos estudios paleoambientales provenientes de Chile central, sur de Mendoza y Neuquén señalan condiciones de estabilidad para el Holoceno tardío, con variaciones menores asociadas a El Niño Oscilación del Sur, semejantes a la actualidad (Markgraf, 1983; Jenny et al., 2002; Whitlock et al., 2006; Le Quesne et al., 2009; Markgraf et al., 2009; Zárate et al., 2010; Bianchi y Ariztegui, 2012; de Jong et al., 2013; Frugone-Álvarez et al., 2020).

En sitios arqueológicos ubicados en ambientes áridos y semiáridos en áreas adyacentes al norte y al sur de $\mathrm{CH} 1$, también se detectaron condiciones de cierta estabilidad en los conjuntos de micromamíferos (e.g., Teta et al., 2005; Fernández et al., 2011a, 2012, 2015a, 2015b, 2016; Fernández, 2012; Pardiñas y Teta, 2013; Fernández y Pardiñas, 2018; López et al., 2021). No obstante, la Cueva Parque Diana, ubicada a $400 \mathrm{~km}$ al sudoeste de $\mathrm{CH} 1$, en un ambiente de bosque cercano a las estepas altoandinas, ofrece un registro de micromamíferos con variaciones en la secuencia temporal (Agnolín y Pérez, 2014). Allí se observan sigmodontinos asociados al bosque (Geoxus valdivianus) o al ecotono bosque-estepa en los niveles más antiguos $(2,35-0,85$ ka cal. AP) y un empobrecimiento taxonómico, posiblemente relacionado con aumentos de la aridez, hacia 0,66-0,55 ka cal. AP. Otro elemento común en los registros de micromamíferos de algunos de estos sitios resulta en el incremento exponencial de las especies de sigmodontinos oportunistas tales como C. musculinus y Oligoryzomys longicaudatus y, en ocasiones, la retracción regional o extinción de especies (e.g., Holochilus spp., Lestodelphys halli) en los conjuntos actuales, que reflejan un deterioro de los ambientes ocasionado, principalmente, por el pisoteo y sobrepastoreo de ovicaprinos ocurridos en las últimas centurias (e.g., Fernández, 2014; Teta et al., 2014; López et al., 2021). Sin embargo, en el área de $\mathrm{CH} 1$ no se detectaron cambios tan marcados ni extinciones recientes de especies, aspecto que sugiere que el impacto antrópico no fue tan significativo.

\section{CONCLUSIÓN}

A partir de la ampliación de la muestra de los micromamíferos y de la mayor resolución arqueológica de $\mathrm{CH} 1$ se alcanzó un conocimiento más 
detallado sobre la configuración de las comunidades de pequeños marsupiales y de pequeños roedores caviomorfos y sigmodontinos que habitaron en las inmediaciones del sitio desde el Pleistoceno tardío final hasta la actualidad.

Al conjunto ya conocido para el sitio, conformado por los roedores Akodon iniscatus, Ctenomys sp., Eligmodontia sp., Euneomys chinchilloides, Microcavia australis y Phyllotis xanthopygus y el marsupial Thylamys pallidior, se adicionan dos nuevos sigmodontinos (Abrothrix olivacea, Oligoryzomys longicaudatus) y un caviomorfo (Galea leucoblephara). Los nuevos datos no favorecen una mayor heterogeneidad ambiental hacia el Holoceno temprano ( 9,5 ka cal. AP), como se había planteado previamente. Por el contrario, permiten inferir una marcada estabilidad del paisaje en general $y$, por lo tanto, de las condiciones climáticas durante el segmento cronológico representado. Se interpreta un ambiente caracterizado por un mosaico de estepas arbustivas, áreas abiertas de suelo desnudo y pedregoso, posiblemente un poco menos árido que en la actualidad por la ausencia de elementos típicos del Monte. Esta estabilidad contrasta con el registro de micromamíferos de otros yacimientos del Pleistoceno tardío/Holoceno de la cuenca superior del río Limay y sugiere respuestas bióticas diferenciales que podrían estar asociadas con la distancia a zonas de englazamiento.

Finalmente, la integración de líneas de evidencia paleoambiental destaca que los eventos climáticos más importantes, a nivel regional, durante el Pleistoceno tardío final (Inversión Climática Huelmo Mascardi) y el Holoceno (neoavances glaciarios), o bien no fueron de suficiente magnitud como para generar modificaciones significativas en las comunidades locales de micromamíferos donde se emplaza $\mathrm{CH} 1$ o no están representados los sedimentos portadores.

\section{Agradecimientos}

A Carlos Cides y Claudia Della Negra, autoridades de Patrimonio Cultural, por las autorizaciones para desarrollar la investigación. La Agencia (PICT 2016-062), el CSIC (i-COOPB-20287) y la UNLP (11/N851) financiaron este trabajo.

\section{REFERENCIAS CITADAS}

Abraham, E., del Valle, H., Roig, F., Torres, L., Ares, J., Coronato, F. y Godagnone, R. (2009). Overview of geography of the Monte Desert biome (Argentina). Journal of Arid Environments, 73, 144-153. https://doi. org/10.1016/j.jaridenv.2008.09.028

Agnolín, F.L. y Pérez, A. (2014). Micromamíferos (Chiroptera-Rodentia) del sitio "Cueva Parque Diana" (provincia de Neuquén, Argentina) y sus implicancias paleoambientales para el Holoceno tardío de Patagonia norte. Historia Natural, Tercera Serie, 4, 5-29.

Andrews, P. (1990). Owls, Caves and Fossils. University of Chicago Press.

Barberena, R. (2014). Discordancias y discontinuidades en Patagonia septentrional: crono-estratigrafía de Cueva Huenul 1 (Neuquén, Argentina). En V. Cortegoso, V. Durán y A. Gasco (Eds.), Arqueología de ambientes de altura en Mendoza y San Juan (pp. 203-219). EDIUNC, Universidad Nacional de Cuyo.

Barberena, R. (2017). Cueva Huenul 1 Archaeological Site (Northwestern Patagonia, Argentina): Initial Colonization and mid-Holocene Demographic Retraction. Latin American Antiquity, 20, 304-318. https://doi.org/10.7183/1045-6635.26.3.304

Barberena, R., Pompei, M., Otaola, C., Gil, A., Neme, G., Borrazzo, K., Durán, V. y Hoguin, R. (2010). Pleistocene-Holocene Transition in Northern Patagonia: Evidences from Cueva Huenul 1 (Neuquén, Argentina). Current Research in the Pleistocene, 27, 5-8.

Barberena, R., Borrazzo, K., Rughini, A., Romero, G., Pompei, M., Llano, C.,...Nella Quiroga, M. (2015). Perspectivas arqueológicas para Patagonia septentrional: sitio Cueva Huenul 1 (provincia del Neuquén, Argentina). Magallania, 43, 1-27.

Barberena, R., Tessone, A., Nella Wuiroga, M., Gordón, F., Llano, C., Gasco, A., Paiva, J. y Ugan, A. (2018). Guanacos y ecología isotópica en el norte del Neuquén: El registro de Cueva Huenul 1. Revista del Museo de Antropología, 11, 7-14. https://doi. org/10.31048/1852.4826.v11.n1.12005

Beltrame, O., Sardella, N., Fugassa, M. y Barberena, R. (2011). Paleoparasitological analysis of rodent coprolites from the archaeological site Cueva Huenul 1, Patagonia (Argentina). Memorias do Instituto Oswaldo Cruz, 107, 604-608. https://doi.org/10.1590/ $\underline{\mathrm{S} 0074-02762012000500006}$

Bernardis, A. (2019). Ensambles de micromamíferos terrestres no-voladores de la región extra-andina de la provincia del Neuquén (Argentina) [tesis doctoral inédita. Universidad Nacional de La Plata] SEDICI. 
Repositorio Digital de la Universidad Nacional de La Plata. https://doi.org/10.35537/10915/87681

Bianchi, M. y Ariztegui, D. (2012). Vegetation history of the Río Manso Superior catchment area, Northern Patagonia (Argentina), since the last deglaciation. The Holocene, 22, 1283-1295. https://doi. org/10.1177/0959683611405083

De Jong, R., Von Gunten, L., Maldonado, A. y Grosjean, M. (2013). Late Holocene summer temperatures in the central Andes reconstructed from the sediments of high-elevation Laguna Chepical, Chile $\left(32^{\circ} \mathrm{S}\right)$. Climate Past, 9, 1921-1932. https://doi:10.5194/cp-9-1921-2013

Espizua, L. (2003). Holocene glaciar fluctuations in the south of Mendoza Andes, Argentina. En G. Casassa, F. Sepúlveda y R. Sinclair (Eds.), The Patagonian Icefields. Series of the Centro de Estudios Científicos (pp. 55-56). Springer. https://doi.org/10.1007/978-1-4615-0645-4_6

Espizua, L. (2005). Holocene glacier chronology of Valenzuela Valley, Mendoza Andes, Argentina. The Holocene, 15, 1079-1085. https://doi. org/10.1191/0959683605hl866rr

Espizua, L. y Pitte, P. (2009). The Little Ice Age glacier advance in the Central Andes $\left(35^{\circ} \mathrm{S}\right)$, Argentina. Palaeogeography, Palaeoclimatology, Palaeoecology, 281, 345-350.https://doi.org/10.1016/j. palaeo.2008.10.032

Fernández, F. (2012). Microvertebrados del Holoceno de sitios arqueológicos en el sur de Mendoza (República Argentina): aspectos tafonómicos y sus implicancias en la subsistencia humana [tesis doctoral inédita. Universidad Nacional de La Plata]. SEDICI Repositorio Digital de la Universidad Nacional de La Plata. https:// doi.org/10.35537/10915/21965

Férnandez, F. (2014). Micromamíferos como indicadores de deterioro ambiental (antrópico) en el sur de Mendoza. En D. Torres (Ed.), Libro de Resúmenes E-ICES 10 (pp. 282-293). Comisión Nacional de Energía Atómica.

Fernández, F. y Pardiñas, U. (2018). Small mammals taphonomy and environmental evolution during Late Pleistocene-Holocene in Monte Desert: the evidence of Gruta del Indio (central west Argentina). Journal of South American Earth Sciences, 84, 266-275. https:// doi.org/10.1016/j.jsames.2018.04.012

Fernández, F., Pardiñas, U., Teta, P. y Barberena, R. (2011a). Environmental stability during the PleistoceneHolocene transition in northwestern Patagonia? The small mammals of Cueva Huenul 1 as evidence. Current Research in the Pleistocene, 28, 154-156.

Fernández, F., Ballejo, F., Moreira, G., Tonni, E. y De Santis, L. (2011b). Roedores cricétidos de la provincia de Mendoza. Guía cráneo-dentaria orientada para su aplicación en estudios zooarqueológicos. Sociedad
Argentina de Antropología; Universitas Sarmiento.

Fernández, F., Del Papa, L., Mange, E., Teta, P., Crivelli Montero, E. y Pardiñas, U. (2016). Human subsistence and environmental stability during the last 2200 years in Epullán Chica cave (northwestern Patagonia, Argentina): a perspective from the zooarchaeological record. Quaternary International, 391, 38-50. https:// doi.org/10.1016/j.quaint.2015.06.013

Fernández, F., Rudzik, M., Neme, G. y De Santis, L. (2015a). Micromamíferos, tafonomía y ambientes durante los últimos 3.800 años a.p. en el valle medio del río Grande, Mendoza, Argentina. Chungara, 47, 267-285.

Fernández, F., Neme, G. y De Santis, L. (2015b). New small mammals record from Arroyo Malo3 archaeological site of Central West Argentina. Taphonomy, paleoenvironments and Human occupations across the Holocene. Archaeofauna, 24, 27-52. https://revistas.uam.es/archaeofauna/issue/ view/616

Fernández, F., Teta, P., Barberena, R. y Pardiñas, U. (2012). Small mammal remains from Cueva Huenul 1, Argentina. Taphonomy and paleoenvironments since the late Pleistocene. Quaternary International, 278, 2231. https://doi.org/10.1016/j.quaint.2012.01.005

Fernández, F., Montalvo, C., Fernández-Jalvo, Y., Andrews, P., y López, M. (2017). A re-evaluation of the taphonomic methodology for the study of small mammal fossil assemblages of South America. Quaternary Science Reviews, 155, 37-49. https://doi. org/10.1016/j.quascirev.2016.11.005

Folguera, A., Bottesi, G., Zapata, T. y Ramo, V. (2008). Crustal collapse in the Andean backarc since $2 \mathrm{Ma}$ Tromen volcanic plateau, Southern Central Andes (36400-37300S). Tectonophysics, 459, 140-160. https:// doi.org/10.1016/j.tecto.2007.12.013

Frugone-Álvarez, M., Latorre, C., Barreiro-Lostres, F., Giralt, S., Moreno, A., Polanco-Martínez, J.,...ValeroGarcés, B. (2020). Volcanism and climate change as drivers in Holocene depositional dynamic of Laguna del Maule (Andes of central Chile - 36 S). Climate of the Past, 16, 1097-1125. https://doi.org/10.5194/ cp-16-1097-2020

Gil, A., Zárate, M. y Neme, G. (2005). Mid-Holocene paleoenvironments and the archeological record of southern Mendoza, Argentina. Quaternary International, 132, 81-94. https://doi.org/10.1016/j. quaint.2004.07.014

Gordón, F., Barberena, R. y Bernal, V. (Eds.) (2017). El poblamiento humano del norte de Neuquén: estado 
actual del conocimiento y perspectivas. Aspha.

Hajdas, I., Bonani, G., Moreno, P. y Ariztegui, D. (2003). Precise radiocarbon dating of late-glacial cooling in mid-latitude South America. Quaternary Research, 59, 70-78. https://doi.org/10.1016/S0033-5894(02)00017-0

Hofreiter, M., Betancourt, J., Pelliza Sbriller, A., Markgraf, V. y Macdonald, H. (2003). Philogeny, diet, and habitat of an extinct Ground Sloth from Cuchillo Curá, Neuquén Province, Southwestern Argentina. Quaternary Research, 59, 364-378. https://doi.org/10.1016/ S0033-5894(03)00030-9

Jenny, B., Valero-Garcés, B., Villa-Martínez, R., Urrutia, R., Geyh, M. y Vei, H. (2002). Early to Mid-Holocene aridity in Central Chile and the Southern Westerlies: The Laguna Aculeo record ( $\left.34^{\circ} \mathrm{S}\right)$. Quaternary Research, 58, 160-170. https://doi.org/10.1006/qres.2002.2370

Le Quesne, C., Acuña, C., Boninsegna, J., Rivera, A. y Barichivich, J. (2009). Long-term glacier variations in the Central Andes of Argentina and Chile, inferred from historical records and tree-ring reconstructed precipitation. Palaeogeography, Palaeoclimatology, Palaeoecology, 281, 334-344. https://doi.org/10.1016/j. palaeo.2008.01.039

Llano, C. y Barberena, R. (2013). Explotación humana de especies vegetales en Patagonia septentrional: el registro arqueobotánico de Cueva Huenul 1 (Provincia de Neuquén, Argentina). Darwiniana Nueva Serie, 1, 5-19. https://doi.org/10.14522/darwiniana.2014.11.496

Llano, C., Sosa, P., Sánchez, C. y Barberena, R. (2019). Arqueobotánica de Cueva Huenul 1 (Neuquén, Argentina): Selección y procesamiento de especies vegetales. Intersecciones en Antropología, 20, 211-223. https://doi.org/10.37176/iea.20.2.2019.446

Llano, C., De Porras, M., Barberena, R., Timpson, A., Beltrame, M. y Marsh, E. (2020). Human resilience to Holocene climate changes inferred from rodent middens in drylands of northwestern Patagonia (Argentina). Palaeogeography, Palaeoclimatology, Palaeoecology, 557, 109894. https://doi.org/10.1016/j. palaeo.2020.109894

López, M., Aguilar, P. y Fernández, F. (2021). Egagrópilas actuales de aves rapaces como herramienta para la generación de modelos paleoambientales en la provincia de Mendoza, Argentina. Mastozoología Neotropical, 28, 1-19. https://doi.org/10.31687/ saremMN.21.28.1.0.16

Lozada, M., Monjeau, J., Heinemann, K., Guthmann, N. y Birney, E. (1996). Abrothrix xanthorhinus. Mammalian Species, 540, 1-6.

Markgraf, V. (1983). Late and postglacial vegetational and paleoclimatic change in subantartic and arid environments in Argentina. Palynology, 7, 43-70. https://doi.org/10.1080/01916122.1983.9989252

Markgraf, V., Whitlock, C., Anderson, R. y García A. (2009). Late Quaternary vegetation and fire history in the northernmost Nothofagus forest region: Mallín Vaca Lauquen, Neuquén Province, Argentina. Journal of Quaternary Science, 24, 248-258. https://doi. org/10.1002/jqs. 1233

Mercer, J. (1982). Holocene glacier variations in Southern South America. Striae, 8, 35-40.

Pardiñas, U. (1999). Los roedores muroideos del Pleistoceno Tardío-Holoceno en la Región Pampeana (sector este) y Patagonia (República Argentina): aspectos taxonómicos, importancia bioestratigráficas y significación paleoambiental [tesis doctoral inédita. Universidad Nacional de La Plata]. SEDICI Repositorio Digital de la Universidad Nacional de La Plata. http:// sedici.unlp.edu.ar/handle/10915/4529

Pardiñas, U. y Teta, P. (2013). Holocene stability and recent dramatic changes in micromammalian communities of northwestern Patagonia. Quaternary International, 305, 127-140. https://doi.org/10.1016/j. quaint.2012.08.001

Pardiñas, U., Teta, P., Cirignoli, S. y Podestá, D. (2003). Micromamíferos (Didelphimorphia y Rodentia) de Norpatagonia Extra Andina, Argentina: taxonomía alfa y biogeografía. Mastozoología Neotropical, 10, 69-113.

Pearson, O. (1987). Mice and the postglacial history of the Traful valley of Argentina. Journal of Mammalogy, 68, 469-478.

Pearson, A. y Pearson, O. (1993). La fauna de mamíferos pequeños de Cueva Traful I, Argentina: pasado y presente. Praehistoria, 1, 211-224.

Pompei, M., Barberena, R., De Porras, M., Borrazzo, K., Rughini, A. y Gil, A. (2012). Late Quaternary Ecosystems and Humans in Northern Patagonia (Neuquén, Argentina). En L. Miotti, M. Salemme, N. Flegenheimer y T. Goebel (Eds.), Late Pleistocene Peopling of Latin America (pp. 187-190). Current Research in the Pleistocene, Texas A \& M University.

Tammone, M., Lacey, E., Hajduk, A., Christie, M. y Pardiñas, U. (2016). The Quaternary record of Euneomys (Mammalia, Rodentia, Cricetidae) from northwestern Patagonia: evidence for regional extinction. Journal of Vertebrate Paleontology, 36, https://doi.org/10.1080/02724634.2016.1212363

Teta, P., Andrade, A. y Pardiñas, U. (2005). Micromamíferos (Didelphimorphia y Rodentia) y paleoambientes del Holoceno tardío en la Patagonia noroccidental extra-andina (Argentina). Archaeofauna, 14, 183-197. 
Nueva evidencia taxonómica de los pequeños mamíferos de la Cueva Huenul 1 (Neuquén... Intersecciones en Antropología 22(2), julio-diciembre. 2021. ISSN-e 1850-373X

Teta, P., Formoso, A., Tammone, M., de Tommaso, D., Fernández, F. y Pardiñas, U. (2014). Micromamíferos, cambio climático e impacto antrópico: ¿Cuánto han cambiado las comunidades del sur de América del Sur en los últimos 500 años? Therya, 5, 7-38. http://dx.doi. org/10.12933/therya-14-183

Whitlock, C., Bianchi, M., Bartlein, P., Markgraf, V., Marlon, J., Walsh, M. y McCoy, N. (2006). Postglacial vegetation, climate, and fire history along the east side of the Andes (lat $41-2.5^{\circ} \mathrm{S}$ ), Argentina. Quaternary Research, 66, 187-201. https://doi.org/10.1016/j. yqres.2006.04.004

Zárate, M., Gil, A. y Neme, G. (2010). Integrando los registros: generalizaciones, alcances y limitaciones. En M. Zárate, A. Gil y G. Neme (Eds.), Condiciones paleoambientales y ocupaciones humanas del centrooeste de Argentina durante la transición PleistocenoHoloceno y Holoceno de Mendoza (pp. 309-330). Sociedad Argentina de Antropología. 
